\title{
Factibilidad técnica y financiera de explotación del cangrejo dorado de Juan Fernández (Chaceon chilensis)
}

\author{
Guillermo Martínez González y Claudio Alvarez Santander \\ Escuela de Ciencias del Mar. Universidad Católica de Valparaíso \\ Casilla 1020, Valparaíso, Chile \\ E-mail: gmartinez@ucv.cl
}

Recibido: 8 marzo 1999; versión corregida: 1 marzo 2000; aceptado: 20 marzo 2000

\begin{abstract}
RESUMEN. Se analizó la factibilidad técnica y financiera que tendría el montaje de una actividad productiva en el archipiélago de Juan Fernández, basada en la explotación del cangrejo dorado (Chaceon chilensis). Para evaluar el proyecto flota-planta, se utilizó un enfoque de tipo sistémico, que permitió estudiar y estructurar esta situación problema. Se consideró factible una producción de 14,4 ton de cangrejo trozado (pinzas y patas), cocido y congelado sin caparazón, en porciones de 1/2 y 3/4 libras; y 33,6 ton de carne de cangrejo, cocida y congelada en bloques de 1 y 2 libras. Para esta producción, la materia prima se obtendría de la actividad extractiva de 10 embarcaciones artesanales, las cuales operarían en forma complementaria o alternativa a la pesca de langosta.

El proyecto es altamente sensible a los precios tanto de mercado de los productos como de playa de la materia prima. La gran proporción de la inversión se debe al capital de trabajo destinado a la compra de materia prima, en valores que permiten al sector artesanal absorber la inversión en innovaciones tecnológicas requeridas para implementar su flota. Los resultados de la evaluación financiera indican $73 \%$ de probabilidad de éxito en la inversión, Tasa Interna de Retorno (TIR) del 49\%, Retorno sobre la Inversión (RSI) de 1,73 y Valor Actual Neto (VAN) de 17.538,6 UF, equivalentes a $\$ 238.963 .970$.
\end{abstract}

Palabras clave: cangrejo dorado, Chaceon chilensis, factibilidad técnica y financiera, archipiélago de Juan Fernández, Chile.

\section{Technical and financial feasibility of the commercial fishery of Juan Fernandez's golden crab (Chaceon chilensis)*}

\begin{abstract}
The technical and financial feasibility of the golden crab fishery (Chaceon chilensis in Juan Fernandez's archipelago was analyzed. To evaluate the investment project, a systemic approach was used to analyze and to structure this situation problem. It was considered feasible an annual production of 14,4 ton of pieces of crab (pincers and legs), cooked and frozen without shell, in portions of 1/2 and 3/4 pounds; and 33,6 ton of crab meat, cooked and frozen in blocks of 1 and 2 pounds. For this production, the raw material would be supply by the extractive activity of 10 artisanal fishing crafts, which would operate in complementary form or alternative to the lobster fishing.

The project is high sensitive to the prices of the products and the prices of the raw material. A great proportion of the investment is due to the work capital dedicated to the raw material purchase it prevails, in values that the fishermen allow to absorb the investment in technological innovations required to prepare the artisanal fishing craft. The results of the financial evaluation indicate that a probability of $73 \%$ of success of the investment would exist, being calculated a IRR of $49 \%$, a ROI of 1,73 and a NPV of $17.538,6 \mathrm{UF}$, equivalent at $\$ 238.963 .970$ chilean pesos.

Key words: golden crab fishery, Chaceon chilensis, technical and financial feasibility, Juan Fernandez archipelago, Chile.

\footnotetext{
* Artículo generado como parte del "Programa de pesca exploratoria y experimental de recursos pesqueros alternativos a la langosta en las islas Robinson Crusoe y Santa Clara" (Proyecto FIP 95/25), financiado por el Fondo de Investigación Pesquera y realizado por la Universidad Católica de Valparaíso.
} 


\section{INTRODUCCIÓN}

En las aguas circundantes del archipiélago de Juan Fernández, compuesto por las islas Robinson Crusoe, Alejandro Selkirk y Santa Clara habitan numerosas especies marinas de interés comercial, entre las que se destacan la langosta (Jasus frontalis), bacalao (Polyprion oxigeneios), vidriola (Seriola peruanus) y breca (Acantholatris gayi), entre otras. Si bien existe una gran diversidad de especies, sólo algunas de ellas son explotadas, ya sea para su comercialización directa o como carnada para la obtención de otros recursos (Arana y Ziller, 1985).

La actividad pesquera se ha centrado principalmente en la extracción de langosta, constituyéndose este crustáceo en el principal soporte de la actividad pesquera y comercial del archipiélago. Sin embargo, los bajos rendimientos de pesca, asociados a problemas en la gestión comercial de este producto, en los últimos años ha provocado algunos inconvenientes a los pescadores del archipiélago, sobre todo en la irregularidad de sus ingresos.

Entre los recursos pesqueros alternativos a la langosta se encuentra el cangrejo dorado de Juan Fernández (Chaceon chilensis), que se ha demostrado que presenta características carneas apropiadas (Cifuentes y Quiñinao, 2000) y niveles de abundancia que permiten se explotación (Arana, 2000b). Por tal razón, se procedió a evaluar la factibilidad técnica y financiera que tendría el montaje de una actividad productiva y comercial basada en este crustáceo, como alternativa u opción complementaria de los recursos actualmente extraídos en el archipiélago.

\section{MATERIALES Y MÉTODOS}

En el desarrollo del estudio de factibilidad se consideraron aspectos tales como: la ubicación geográfica insular, disponibilidad de un medio de transporte regular y constante, idiosincrasia de los agentes involucrados y, finalmente, la realidad de la estructura comercial-productiva de la isla (Sapag y Sapag, 1993). De igual modo, se analizó la definición del proceso productivo conveniente de realizar en el archipiélago para esta eventual nueva actividad. La etapa primaria de extracción se efectuará allí; no obstante, la etapa secundaria de procesamiento y la terciaria de distribución y comercialización del producto final, se identifica si conviene o no cubrirlas; o bien si es favorable realizarlas parcial o íntegramente.
Para la evaluación de la factibilidad técnica y financiera de la explotación de este recurso por parte de pescadores artesanales de la isla Robinson Crusoe, se utilizó un enfoque de tipo sistémico a fin de analizar y estructurar la situación problema, considerando que las opciones a evaluar cubren una amplia gama de posibilidades, tanto desde el punto de vista de los componentes como de los procesos del sistema productivo (Sapag y Sapag, 1993; Weston y Copeland, 1988). De acuerdo a lo anterior, se formuló un modelo matemático correspondiente a una representación idealizada de la realidad. Su propósito es proporcionar un medio que permita estudiar el comportamiento del sistema pesquero local, con el fin de evaluar la mejor alternativa de explotación de este cangrejo. Además, permitió definir la estructura ideal del sistema productivo, indicando las principales relaciones funcionales entre sus elementos.

A la luz de lo expuesto y concibiendo la actividad pesquera como un sistema variable y dinámico, se estableció un modelo para el análisis del sistema de explotación del recurso cangrejo dorado de Juan Fernández, que interrelaciona tanto factores externos como internos del sistema pesquero (Fig. 1).

\section{Consideraciones para la evaluación}

\section{Factores exógenos}

En primer lugar, se considera la potencialidad en función de la abundancia y facilidades, en términos de distribución que presenta el recurso seleccionado, así como la factibilidad de implementación del arte e innovación tecnológica requerida en las embarcaciones para la explotación del recurso. Esto derivado de los resultados obtenidos en la pesca exploratoria con trampas realizadas alrededor de las islas Robinson Crusoe y Santa Clara (Arana, 2000a) y experimental del cangrejo dorado (Arana y Vega, 2000), que establecen la posibilidad de realizar la etapa primaria en ese archipiélago.

La existencia de una pesquería tradicional y firmemente asentada en el archipiélago, como la de langosta, limita el número de embarcaciones posibles de derivar a la pesquería alternativa. Por esta razón, se estima que un máximo del $50 \%$ de la flota operante podría trasladar su esfuerzo al cangrejo dorado de profundidad de enero a octubre y el $50 \%$ restante se adicionaría a la actividad entre mediados de mayo y principios de octubre, en la época de veda de la langosta. 


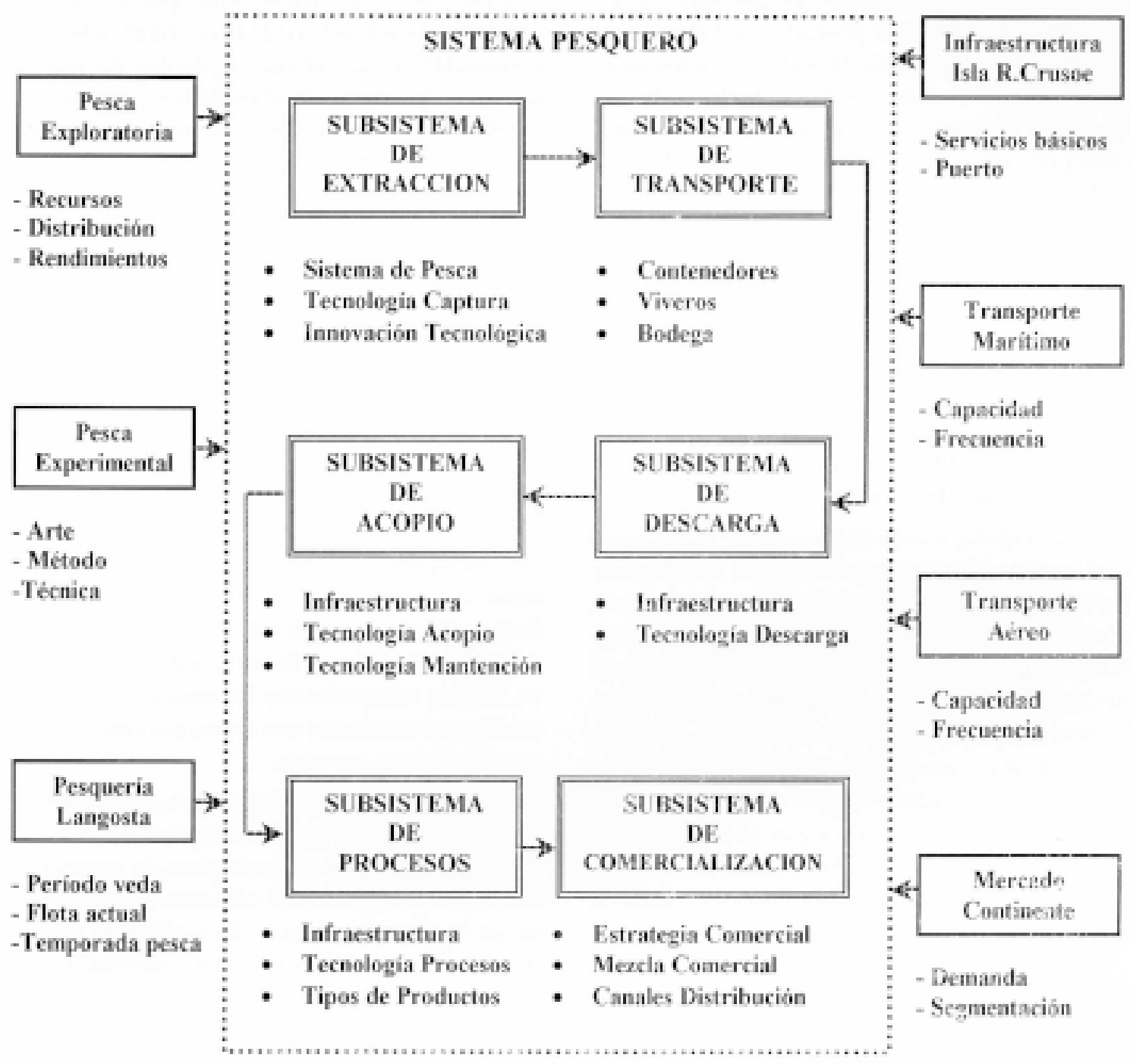

Figura 1. Modelo general de análisis del sistema de explotación del cangrejo dorado de Juan Fernández. Figure 1. Systemical approach model of the Juan Fernandez's golden crab fishery. 
No obstante lo anterior, dadas las malas condiciones meteorológicas reinantes en la zona durante el otoño e invierno, se supone una reducción meteorológica que equipararía la potencialidad de la flota durante esa época, manteniendo los rendimientos de pesca con respecto al período en que sólo opera el $50 \%$ de la flota sobre la pesquería de cangrejo. Se considera adicionalmente, que todas las embarcaciones disponibles actuarían sobre la langosta durante los meses noviembre y diciembre, dadas las condiciones de mercado que hacen irremplazable la actividad en ese período.

Por otro lado, se verifica que la isla Robinson Crusoe no posee abastecimiento suficientemente regular y constante de energía eléctrica para un emplazamiento industrial $\mathrm{y}$, adicionalmente, no presenta condiciones que faciliten la implementación de sistemas de alcantarillado y eliminación de desechos. Sin embargo, en términos de infraestructura, los servicios de puerto y muelle permiten el desarrollo de una actividad sostenida y estable.

Con respecto a la comunicación de la isla con el continente, se debe mencionar que las vías de transporte tanto marítima como aérea no son periódicas ni continuas, por lo que se ven restringidas las posibilidades de transporte sistemático del producto final hacia los centros de consumo en el continente. Es necesario resaltar que las islas Robinson Crusoe y Santa Clara se encuentran separadas del continente por 365 millas náuticas, aproximadamente. Esto implica que para llegar con algún tipo de producto desde ese lugar al mercado consumidor, se debe franquear necesariamente una extensa ruta a través de algún medio de transporte adecuado.

\section{Factores internos}

Con respecto al subsistema de extracción y transporte, que las embarcaciones artesanales actuales están en condiciones de ser derivadas a la pesquería de cangrejo dorado, ya que este recurso soporta el transporte a bordo y sólo se requiere de manipulación adecuada y la existencia de las condiciones de hidratación menores para su mantención en vivo (Arana, 2000). Por esta razón, no se necesitan sistemas especializados de transporte de la captura, después de las faenas de pesca.

Por otro lado, los subsistemas de acopio, procesos y comercialización se ven ligados, ya que las características carneas del recurso le permiten una vida útil después de la muerte de un máximo de seisdías pese a ser sometido a cocción (Cifuentes y Quiñinao,
2000). Aun cuando se mantuviese refrigerado, dadas las características de transporte disponibles ya mencionadas, se hace obligatorio derivar la pesca o a consumo directo por los habitantes de la isla, a mantención de los ejemplares en viveros en espera de transporte que permita la comercialización del recurso vivo en el continente, o a una etapa secundaria que le otorgue por una parte mayor valor agregado al producto $\mathrm{y}$, por otra, una viabilidad de mantención por mayor tiempo como producto congelado.

Con relación al tipo de transporte entre el archipiélago y el continente, existen dos opciones: vía marítima y vía aérea. El medio aéreo dispone de una pista de tierra donde pueden aterrizar aviones de pequeña capacidad, sin que estas aeronaves puedan superar los $600 \mathrm{~kg}$. de carga. Por otro lado, el enlace marítimo es factible de realizar gracias a un muelle de reciente construcción; sin embargo, de las embarcaciones que actualmente realizan viajes hacia la isla, sólo una cuenta con sistemas de refrigeración para la preservación de los productos y posee viveros capaces de transportar los ejemplares vivos hasta el continente. Esta embarcación además se dedica a la comercialización de langosta por lo que es factible de derivar a la nueva actividad productiva, cuando menos durante los meses de veda, para así llegar al continente con aproximadamente 2.500 cangrejos vivos por viaje.

Adicionalmente, considerando los volúmenes de captura de cangrejo posibles de generar con la actividad pesquera, la escasez de plaza de mercado en la isla y las costumbres de consumo en el continente, se ha resuelto como vía realista el evaluar la factibilidad de generar productos congelados a partir del cangrejo dorado, procesado en origen en una planta ubicada en la isla Robinson Crusoe. El o los productos obtenidos se destinaría hacia el mercado internacional, de acuerdo a la existencia de símiles ya establecidos con importancia económica.

\section{RESULTADOS Y ANÁLISIS}

\section{Localización}

En base a las consideraciones planteadas anteriormente y definidas las fases del proceso productivo a desarrollar en las islas, es necesaria la instalación de infraestructura terrestre para realizar actividad productiva y comercial derivada de la explotación del recurso. Esta infraestructura se debe ubicar en la isla Robinson Crusoe, único lugar con habitantes y con instalaciones portuarias aptas para 
el desembarque, donde se centralizan las actividades realizadas por los pescadores artesanales del archipiélago. En efecto adyacente al muelle se encuentran dos caletas donde son varados los botes pesqueros, como también instalaciones que brindan comodidades y servicios directos a los pescadores, como Capitanía de Puerto, carpintería naval, bencineras, y casetas o boxes para guardar los materiales, equipos e insumos de pesca.

En la actualidad, en la isla no existe un plano regulador que permita identificar una zona para el emplazamiento industrial; no obstante, de acuerdo a lo informado por las actuales autoridades de la comuna, es posible ubicar el proyecto en las cercanías del poblado. Por otra parte, en la isla Robinson Crusoe se ha solucionado el problema de abastecimiento de agua potable, proporcionándose en forma regular y suficiente, permitiendo el funcionamiento permanente de plantas procesadoras de alimentos que utilizan en forma abundante este elemento.

Si bien la isla existe el servicio de energía eléctrica, este suministro se debe asegurar incorporando un grupo electrógeno en la inversión inicial de la planta, de acuerdo a los requerimientos de potencia de la totalidad del equipamiento requerido para el proceso. Se recomienda localizar la planta en un lugar que posea a lo menos $400 \mathrm{~m} 2$, que presente posibilidades para futuras ampliaciones y que permita una comunicación expedita con el puerto de desembarque de la materia prima.

\section{Ingeniería}

En esta etapa se resuelve el problema técnico, presentándose las alternativas viables, satisfactorias y más favorables para realizar una actividad productiva comercial con el recurso cangrejo dorado. Como en la mayoría de los proyectos pesqueros, esta fase consta de dos aspectos: ingeniería de flota e ingeniería de planta. La ingeniería de flota incluye a su vez, dada la ubicación geográfica del archipiélago, un centro de acopio donde se puedan acumular las capturas desembarcadas. Ello permitirá mantenerlas por tiempos prolongados y en cantidades suficientes como para lograr cargamentos completos para el medio de transporte seleccionado, asegurando el mantenimiento del recurso en excelentes condiciones de conservación, independiente de las oportunidades que existan de transportar el producto al continente. En segundo término, se plantea la ingeniería de planta como opción viable en la búsqueda de productos con mayor valor agregado e interés comercial.

Para ello, se consideraron los resultados obtenidos por Arana (2000b), referente a estimaciones de abundancia del recurso, rendimiento de captura, tecnología extractiva, abastecimiento de materia prima, zonas de captura, métodos y equipos de pesca, y modificaciones que se deben realizar a las embarcaciones locales para la explotación pesquera. Además, Arana y Vega (2000) define en forma específica los regímenes de operación adecuados para la explotación del recurso.

Por otro lado, Cifuentes y Quiñinao (2000) entregan antecedentes sobre la manipulación, tratamieno y preservación del cangrejo dorado, como también sobre los productos obtenidos a través de su procesamiento, con la finalidad de establecer los requerimientos de las instalaciones en tierra.

De acuerdo a lo ya señalado, se estableció para la evaluación económica a nivel de anteproyecto un modelo de la factibilidad de la explotación comercial del recurso cangrejo dorado de Juan Fernández que optimiza la interrelación entre los sistemas flota pesquera y planta de proceso tomando en cuenta tanto las inversiones en innovación tecnológica como los costos de la operación (Fig. 2).

\section{Ingeniería de flota}

Descripción del régimen seleccionado: En base a los resultados de la pesca experimental obtenidos por Arana y Vega (2000), se determinaron 3 regímenes operacionales para la pesca del cangrejo dorado. Estos regímenes son similares entre sí y dependen de la profundidad de calado de las trampas $(200,400$ y 600 metros), difiriendo levemente sólo en los tiempos de virado y calado. En vista de ello, como régimen tipo se estableció aquel realizado a una profundidad de calado de 400 metros.

La duración de la salida permite realizar una marea diaria, en la que se calan 5 líneas con 2 trampas cada una, siendo el rendimiento estimado por trampa para este régimen de 6 cangrejos, lo que permite proyectar una captura de 60 ejemplares/día por bote operativo. El tiempo de navegación total de la salida de pesca es de $140 \mathrm{~min}$, por lo que al considerar el consumo promedio de combustible de las embarcaciones de 1,30 1/hr y las actividades de virado del arte, se estima un consumo total de entre 15 y 17 litros por salida. 


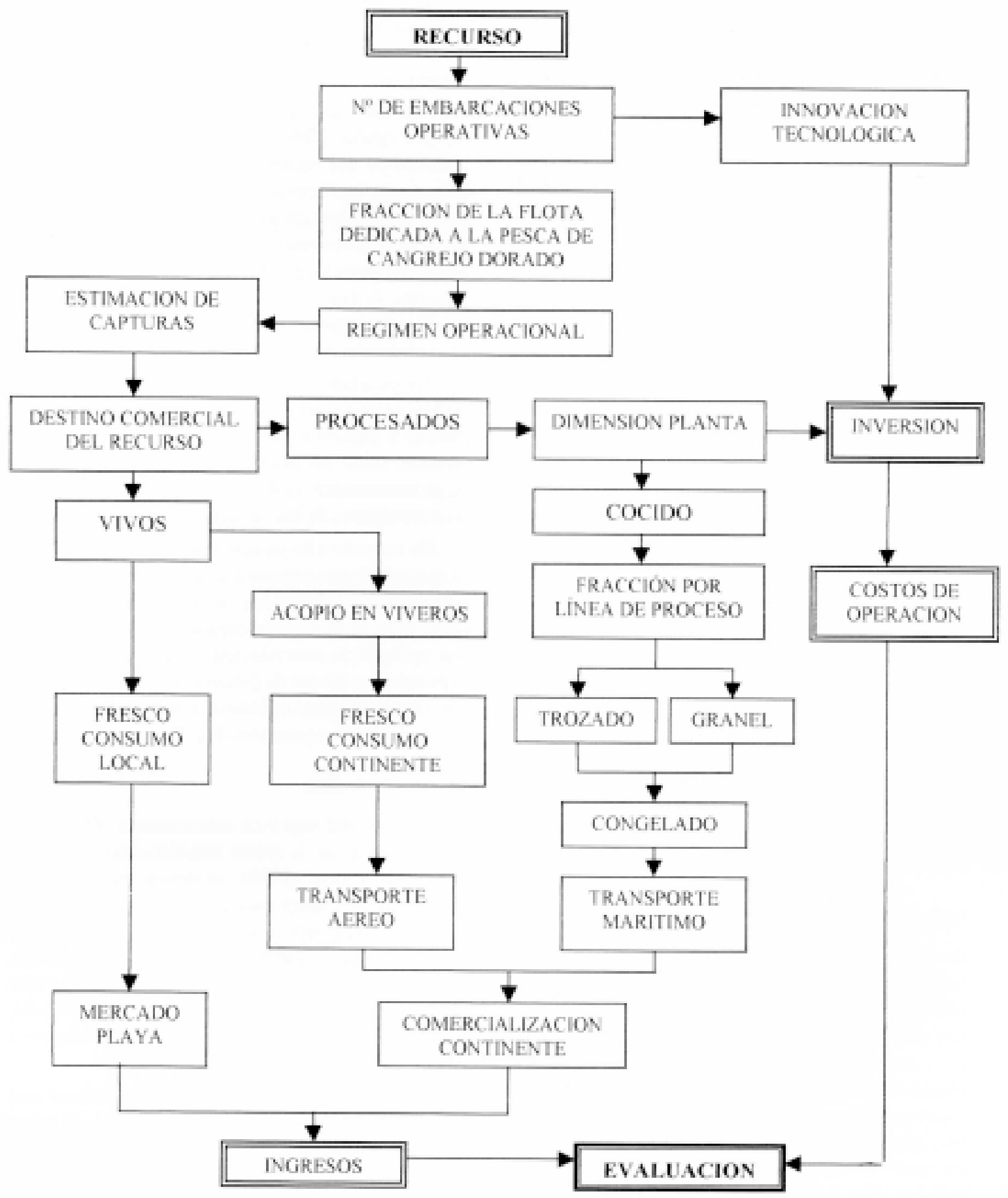

Figura 2. Modelo general de evaluación de la factibilidad técnica y financiera de la explotación comercial del cangrejo dorado en Juan Fernández.

Figure 2. Assessment model of the technical and financial feasibility of the commercial fishery of Juan Fernandez' golden crab. 
Dado que con la muerte del cangrejo comienza el desarrollo de una serie de alteraciones de naturaleza enzimática, química y microbiológica, se hace necesario mantenerlo vivo hasta su procesamiento. Los cambios en la carne son irreversibles, alterándose su textura, apariencia y sabor; además, se produce una disminución en el rendimiento de carne por adhesión de ésta con la caparazón. Por esto, las embarcaciones deben poseer condiciones de transporte y preservación adecuadas, para que el cangrejo pueda permanecer vivo por mayor tiempo. En vista de ello, los ejemplares deben ser almacenados en un ambiente húmedo, en zonas de la embarcación donde sea factible protegerlos, cubriéndolos con material húmedo como géneros capaces de retener humedad.

Innovación tecnológica: En la pesca experimental, se determinó que la trampa es el aparejo de pesca más adecuado para la captura de esta especie (Arana y Vega 2000). Por ello, y de acuerdo al régimen operacional ya especificado, las embarcaciones artesanales requerirían incorporar los siguientes elementos tecnológicos para su normal operación:

- Plato virador hidráulico vertical montado sobre bancada o pescante.

- Sistema hidráulico compuesto por: motor de combustión interna de 7 a 10 HP, bomba hidráulica, estanque de almacenamiento de aceite hidráulico con filtro de aceite, válvula de control de flujo, mangueras y fitting de alta y baja presión.

- Pescante con pasteca, en caso de instalar virador sobre la bancada.

Este equipamiento requiere una inversión referencial de \$1.600.000; por lo que considerando un precio de venta estimado de $\$ 500$ por ejemplar capturado, en las condiciones de operación antes señaladas, la inversión sería recuperada durante el primer año de operación. Cabe señalar, que este precio de playa fue calculado de acuerdo a los ingresos requeridos para la marginación de la pesca de langosta desde un punto de vista del costo de oportunidad alternativo de la actividad.

Integración del subsistema de descarga y acopio: Actualmente, en la isla Robinson Crusoe el desembarque de productos pesqueros se realiza gracias a la existencia de un muelle de reciente construcción, adecuado para esta labor. Para el acopio del cangrejo vivo, se pueden utilizar viveros flotantesde características similares a los ya ocupado por la actividad langostera, estimándose cargas de hasta 200 ejemplares en cada uno de ellos.

$\mathrm{Si}$ bien es cierto que esta primera fase no presenta problemas, la real factibilidad de comercializar el recurso vivo en el continente es escasa, dado que el costo del transporte aéreo no permite generar márgenes de ganancia suficiente, y por otro lado, el transporte marítimo es por el momento posible a través de un ambarcación que posse viveros, cuyo uso durante casi todo el tiempo está destinado al transporte de langostas. Es por eso, que si bien se puede implementar un sistema de acopio de cangrejos en viveros, se sugiere y a la vez se asume para el dimensionamiento de la planta, un destino de la captura en un $100 \%$ al proceso de congelados.

\section{Ingeniería de planta}

Rendimientos del proceso: En términos comparativos, los rendimientos que presenta el cangrejo dorado son muy similares a los registrados en jaiba mora (Homalaspis plana), destacándose el aprovechamiento de las pinzas del recurso y el peso inicial que ellas presentan (Cifuentes y Quiñinao, 2000) (Tabla I).

Tabla 1. Rendimientos de proceso del cangrejo dorado de Juan Fernández. Table 1. Yields of the processing of Juan Fernandez's golden crab.

\begin{tabular}{|lccc|}
\hline Zona del cangrejo & Peso inicial $(\mathbf{g})$ & Peso final $(\mathrm{g})$ & \% Rendimiento \\
\hline Pinzas (c/u) & 97,40 & 35,65 & 36,60 \\
Patas (c/u) & 33,20 & 10,60 & 31,90 \\
Caparazón & 562,80 & 102,60 & 18,00 \\
Rendimiento Total & $1.033,20$ & 258,70 & 25,08 \\
\hline
\end{tabular}

Fuente: (Cifuentes y Quiñinao, 2000). 
Selección de productos: De acuerdo a las características proximales de la materia prima establecidas por Cifuentes y Quiñinao (2000), se ha considerado como punto de referencia los productos comúnmente fabricados por la industria nacional dedicada a jaiba mora. A partir de estas opciones, dadas las condicionantes tanto de infraestructura disponible en la isla Robinson Crusoe, como los escasos medios de transporte existentes y las características de mercado respecto a símiles de la especie seleccionada los productos considerados en este proyecto son:

- Cangrejo trozado: pinzas y patas cocidas y congeladas sin caparazón. Se presenta en bolsa de 0,6 micras selladas al vacío con $1 / 2$ y $3 / 4 \mathrm{lb}$ de contenido neto, embaladas en cajas térmicas de polietileno expandido de $40 \mathrm{lb}$ de copntenido neto.

- Carne de cangrejo: cocida, decorada y congelada en bloques de 1 y $2 \mathrm{lb}$, envasadas en bolsas de polietileno atmósfera normal y agrupadas en cajas térmicas de $40 \mathrm{lb}$ de contenido neto.

- Cangrejo entero fresco: no requiere proceso en planta.

Determinación de escalas de producción: Para determinar las escalas de producción, se han considerado los siguientes aspectos:

- Dedicación a la explotación del cangrejo dorado de un máximo del $50 \%$ de la flota artesanal de la isla,considerando que la fracción restante se mantiene en la actividad actual correspondiente a la pesquería de la langosta.

- El régimen operacional seleccionado no es compatible con el corespondiente a la pesquería de langosta por lo que se considera de dedicación exclusiva.

- Durante la temporada de veda de la langosta, la flota se dedicaría en un $100 \%$ a la pesquería de cangrejo. Sin embargo, dadas las condiciones climáticas del archipiélago durante esa época, se asume una reducción meteorológica del $50 \%$ lo que permite mantener el nivel de producción de la flota durante el año.

- Dada las condiciones tradicionales de la actividad desarrollada por los pescadores artesanales y las características de mercado hacia final de año, se emplea la dedicación del $100 \%$ de la flota a la pesquería de langosta durante los meses de noviembre y diciembre.

- Para el cálculo de las escalas de producción de la planta se considera la condición máxima de producción en que el $100 \%$ de las capturas realizadas por la flota artesanal es derivada a la planta, asignándose la materia prima en fracciones iguales a las dos líneas de proceso.

- En la operación de pesca se consideran situaciones: pesimista (rendimiento 4 ejemplares por trampa), normal (6 ejemplares por trampa) y optimista ( 8 ejemplares por trampa), para 20,15, 10 y 5 botes de dedicados a la pesquería de cangrejo de profundidad, con 200,150, 100 y 50 trampas totales, respectivamente. De acuerdo a esto, y considerando un peso promedio de un kilo por ejemplar, la captura total anual en toneladas realizada por flota a distintas escalas, sería la siguiente:

\begin{tabular}{|cccc|}
\hline $\begin{array}{c}\text { Número de } \\
\text { embarcaciones }\end{array}$ & $\begin{array}{c}\mathbf{4} \mathbf{~ k g} / \\
\text { trampa }\end{array}$ & $\begin{array}{c}\mathbf{6} \mathbf{~ k g} / \\
\text { trampa }\end{array}$ & $\begin{array}{c}\mathbf{8} \mathbf{~ k g} / \\
\text { trampa }\end{array}$ \\
\hline $\mathbf{5}$ & 30 & 45 & 60 \\
$\mathbf{1 0}$ & 60 & 90 & 120 \\
$\mathbf{1 5}$ & 90 & 135 & 180 \\
$\mathbf{2 0}$ & 120 & 180 & 240 \\
\hline
\end{tabular}

A la luz de las condiciones ya presentadas, adicionando la realidad del archipiélago y una proyección sostenida de la actividad sin comprometer la dedicación y actividad actual de la flota artesanal, se ha determinado que el dimensionamiento de la planta se debería efectuar a partir de la operación inicial de 10 embarcaciones $(25 \%$ de la flota operativa actual).

Procesamiento de los cangrejos: Los cangrejos capturados por la flota artesanal son almacenados en viveros en la fase de acopio; luego, son llevados hacia planta en bins para ingresar posteriormente a las líneas de proceso. El producto final, luego de cualquiera de los procesos efectuados, es almacenado en una cámara de frío a $-20^{\circ} \mathrm{C}$ hasta el momento de efectuar los envíos al continente.

Maquinaria, equipos y materiales principales y secundarios: El proceso de congelado a partir de cangrejo cocido, requiere de 15 a 16 etapas según la línea de elaboración desarrollada, para las cuales se requieren maquinarias, equipos y materiales principales que en ciertas fases del proceso son comunes. En función de lo anterior y del cumplimiento de los requerimientos básicos del proceso, se seleccionaron los elementos que conforman el equipamiento de planta. Del mismo modo, dicho proceso requiere de materiales e 
insumos secundarios cuya cantidad fue definida de acuerdo a la cantidad de materia prima ingresada a planta, rendimientos y secuencias productivas seleccionadas.

Servicios: Los servicios requeridos para este proyecto son electricidad y agua potable. Como la energía eléctrica no es proporcionada en forma segura y continua en la isla Robinson Crusoe, se considera tener autonomía invirtiendo en un grupo electrógeno de 100 $\mathrm{kw} / \mathrm{hr}$. El costo de funcionamiento de este equipo, de acuerdo a la energía requerida para la marcha de la planta, es asignado al costo en servicio eléctrico correspondiente en que se incurrirá. Adicionalmente, con relación al suministro de agua potable, se incluye el montaje de un equipo Hidropack que asegure la presión de agua requerida para el correcto funcionamiento de la planta. Los requerimientos de servicios para el funcionamiento de la planta son:

\section{Electricidad $\quad 29,71 \mathrm{Kw} / \mathrm{hr}$ Agua potable $\quad 9,31 \mathrm{~m}^{3} /$ día}

Lay-out, áreas de planta y edificaciones: La Fig. 3 muestra el lay-out de la planta. El área requerida para la planta de proceso y edificaciones es la siguiente:

$\begin{array}{lr}\text { Area de planta } & 300 \mathrm{~m}^{2} \\ \text { Oficinas } & 18 \mathrm{~m}^{2} \\ \text { Vestidores, duchas y } & \\ \text { servicios higiénicos } & 40 \mathrm{~m}^{2}\end{array}$

Obras civiles, instalación y montaje: La ejecución de las obras civiles, instalación y montaje de la planta y todos sus equipos, se recomienda sea realizada por personal especializado llevado a la isla Robinson Crusoe desde el continente, al igual que todos los materiales requeridos para la construcción. Esto, dadas las condiciones de indisponibilidad que presentan actualmente la isla; por lo que se incluyó al costo de construcción el transporte y estadía de los elementos $\mathrm{y}$ agentes involucrados.
Operación de la planta: Finalizada la fase de construcción y puesta en marcha, la planta podrá comenzar a operar a un $100 \%$ de su capacidad de producción. En condiciones normales, se contempla una operación continua durante 200 días al año, sin considerar los meses de máxima demanda de langosta (noviembre y diciembre), con jornadas de trabajo de 8 horas por diarias, tiempo en cual es posible producir anualmente 14,4 ton de cangrejo trozado y 33,6 ton de carne de cangrejo.

Requerimientos de personal: De acuerdo al volumen de producción estimado, para el normal funcionamiento de la planta se requiere contar con un jefe de planta, un funcionario administrativo, un calderero y diez operarios, lo que reporta un total de trece personas.

\section{Estudio de tamaño}

Como este proyecto es de línea semi-industrial, se ha adoptado dimensionar su tamaño considerando la capacidad de producción normal en un período de tiempo dado. La producción se determinó sobre la base de la disponibilidad de materia prima en la pesquería durante el año, que comprende una temporada de 10 meses, de enero a octubre.

Las escalas de producción de la planta, se calculan en condición máxima, es decir, cuando la totalidad de las capturas realizadas por la flota artesanal son entregadas a la planta, asignándose la materia prima en fracciones iguales a las dos líneas de proceso.

En la definición del tamaño del proyecto existe un rango de variación de acuerdo a los rendimientos obtenidos por trampa y al número de embarcaciones dedicadas a la pesquería, obteniéndose en función de esto las siguientes producciones (ton/año): 


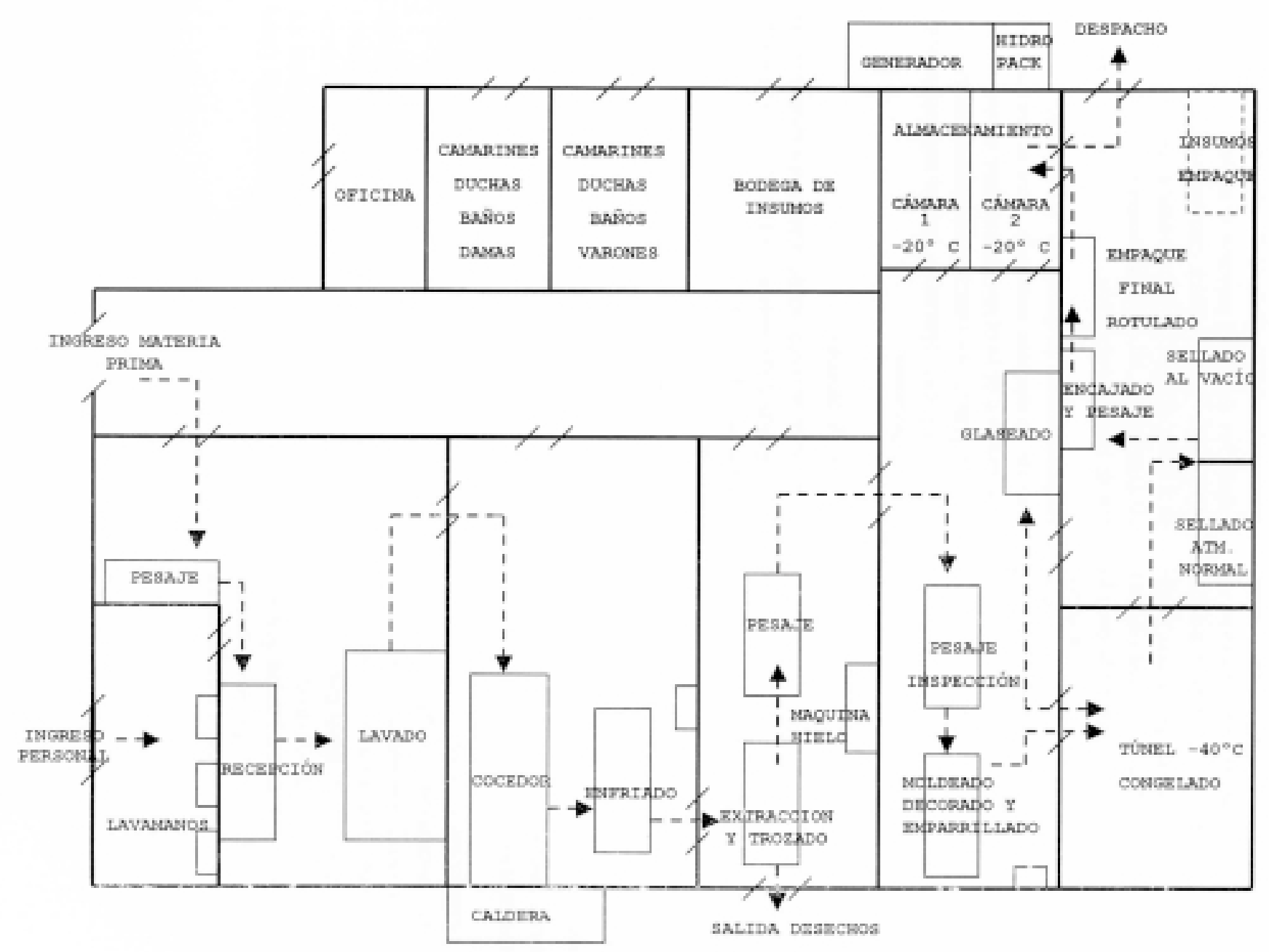

Figura 3. Lay out de la planta propuesta.

Figure 3. Lay out ot fhe proposal frozen plant. 
Proyección optimista de producción anual en ton (rendimiento 8 kg/trampa):

\begin{tabular}{|ccccc|}
\hline Línea de proceso/Dedicación flota & 5 botes & 10 botes & 15 botes & 20 botes \\
Trozado & 9,0 & 18,0 & 27,0 & 36,0 \\
Bloques & 13,8 & 42,0 & 63,0 & 84,0 \\
Total & 22,8 & 60,0 & 90,0 & 120,0 \\
\hline
\end{tabular}

Situación normal de producción anual en ton (rendimiento 6 kg/trampa):

\begin{tabular}{ccccc}
\hline Línea de proceso/Dedicación flota & 5 botes & 10 botes & 15 botes & 20 botes \\
Trozado & 7,20 & 14,4 & 21,6 & 28,8 \\
Bloques & 11,04 & 33,6 & 50,4 & 67,2 \\
Total & 18,24 & 48,0 & 72,0 & 96,0
\end{tabular}

Proyección pesimista de producción anual en ton (rendimiento 4 kg/trampa):

\begin{tabular}{|clclc|}
\hline Línea de proceso/Dedicación flota & 5 botes & 10 botes & 15 botes & 20 botes \\
Trozado & 5,4 & 10,8 & 16,2 & 21,6 \\
Bloques & 12,6 & 25,2 & 37,8 & 50,4 \\
Total & 18,0 & 36,0 & 54,0 & 72,0 \\
\hline
\end{tabular}

\section{Análisis económico y financiero}

Estimación de capital de trabajo

Para estimar el capital de trabajo se estimó una actividad inicial de tres meses. Este incluye el costo de materia prima, insumos, servicios y personal, llegándose a un total de $\$ 34.785 .286$, lo que equivale a $\$ 13.261 .762$ mensuales. La materia prima es el ítem más relevante, representando un $72,4 \%$ del total.

\section{Materia prima:}

Cangrejo dorado de Juan Fernández (Ejemplares)

Subtotal 1 Capital de trabajo

\section{Cantidad \$/Unidad Total}

57.600

$\$ 500 \$ 28.800 .000$

$\$ 28.000 .000$

\section{Insumos:}

Bolsas Dinoseal

Etiquetas para bolsas

Bolsas para sellado (Contenido $1 \mathrm{lb}$ )

Bolsas para sellado (Contenido $2 \mathrm{lb}$ )

Etiquetas para bolsas

Cajas de polietileno expandido para $40 \mathrm{lb}$

Rótulos de despacho

Cinta para sellado (rollo)

Bolsas cubierta cajas

Solución clorada (l)

Solución yodada (1)

Ropa de trabajo

Subtotal 2 Capital de Trabajo
Cantidad \$/Unidad Total

$\begin{array}{rrr}6.067 & \$ 342 & \$ 2.075 .056 \\ 6.067 & \$ 4 & \$ 24.270 \\ 11.077 & \$ 20 & \$ 221.538 \\ 5.538 & \$ 20 & \$ 110.769 \\ 16.615 & \$ 4 & \$ 66.462 \\ 791 & \$ 2.310 & \$ 1.827 .692 \\ 791 & \$ 4 & \$ 3.165 \\ 40 & \$ 350 & \$ 13.846 \\ 791 & \$ 20 & \$ 15.824 \\ 300 & \$ 700 & \$ 210.000 \\ 180 & \$ 650 & \$ 117.000 \\ - & - & \$ 52.600 \\ & & \$ 4.738 .223\end{array}$




\begin{tabular}{|c|c|c|c|}
\hline Servicios: & Cantidad & \$/Unidad & Total \\
\hline Electricidad $(\mathrm{Kw})$ & $19.489,8$ & $\$ 49$ & $\$ 954.999$ \\
\hline Agua $\left(\mathrm{m}^{3}\right)$ & 558,6 & $\$ 236$ & $\$ 132.064$ \\
\hline Subtotal 3 capital de trabajo & & & $\$ 1.087 .063$ \\
\hline Fuerza laboral: & Cantidad & $\$ / \mathbf{m e}$ & Total \\
\hline Jefe de planta & 1 & $\$ 250.000$ & $\$ 750.000$ \\
\hline Calderero & 1 & $\$ 150.000$ & $\$ 450.000$ \\
\hline Secretaria & 1 & $\$ 120.000$ & $\$ 360.000$ \\
\hline Operarios & 10 & $\$ 120.000$ & $\$ 3.600 .000$ \\
\hline Subtotal 4 capital de trabajo & & & $\$ 5.160 .000$ \\
\hline Total capital trabajo & & & $\$ 39.785 .286$ \\
\hline
\end{tabular}

En función de las características de las líneas de elaboración seleccionadas y los volúmenes de captura proyectados para la condición de evaluación, la inversión requerida en maquinarias, equipos, infraestructura y montaje de las instalaciones alcanza la suma de \$83.396.042. Este monto se detalla a continuación:

\begin{tabular}{|lrrr|} 
Maquinaria y equipos: & Cantidad & $\$ /$ unidad & Total $(\$)$ \\
\hline Balanza recepción & 1 & $\$ 768.000$ & $\$ 768.000$ \\
Máquina productora de hielo & 1 & $\$ 1.500 .000$ & $\$ 1.500 .000$ \\
Cámara de mantención 1 & 1 & $\$ 2.900 .000$ & $\$ 2.900 .000$ \\
Cámara de mantención 2 & 1 & $\$ 2.900 .000$ & $\$ 2.900 .000$ \\
Túnel de congelación & 1 & $\$ 7.000 .000$ & $\$ 7.000 .000$ \\
Balanza proceso & 1 & $\$ 313.950$ & $\$ 313.950$ \\
Selladora al vacío & 1 & $\$ 2.301 .000$ & $\$ 2.301 .000$ \\
Selladora & 1 & $\$ 300.000$ & $\$ 300.000$ \\
Hidropack & 1 & $\$ 1.550 .000$ & $\$ 1.550 .000$ \\
Generador eléctrico & 1 & $\$ 15.000 .000$ & $\$ 15.000 .000$ \\
Cocedor & 1 & $\$ 1.800 .000$ & $\$ 1.800 .000$ \\
Caldera & 1 & $\$ 6.200 .000$ & $\$ 6.200 .000$ \\
Subtotal 1 Maquinaria y Equipos & & & $\$ 42.532 .950$ \\
\hline
\end{tabular}

Materiales:

Subtotal 2 Materiales

$\$ 10.733 .092$

Instalaciones:

Total

Terreno
Compra y construcción galpón
Construcción oficinas vestuarios y baños
Red eléctrica e instalación
Red suministro de agua e instalación
Red de vapor
Subtotal 3 Instalaciones

$(*)$

$\$ 22.500 .000$

$\$ 3.000 .000$

$\$ 2.660 .000$

$\$ 1.800 .000$

$\$ 170.000$

$\$ 30.130 .000$

Inversión Total $\$ 83.396 .042$

(*): El terreno sería entregado en comodato por la Ilustre Municipalidad de Juan Fernández. 


\section{Requerimiento de capital}

La inversión requerida en planta, considerando construcción, maquinarias, equipos, materiales, capital de trabajo e interés por el financiamiento, es de \$137.882.029; la cual se detalla a continuación:

\begin{tabular}{|c|c|}
\hline Costo construcción de la planta & $\$ 30.130 .000$ \\
\hline Costo maquinaria y equipos & $\$ 42.532 .950$ \\
\hline Costo materiales & $\$ 10.733 .092$ \\
\hline Subtotal & $\$ 83.396 .042$ \\
\hline Capital de trabajo & $\$ 39.785 .286$ \\
\hline Subtotal & $\$ 123.181 .328$ \\
\hline Interés & $\$ 14.700 .701$ \\
\hline Total & $\$ 137.882 .029$ \\
\hline
\end{tabular}

Evaluación financiera

Condiciones:

- El horizonte del proyecto se consideró en 15 años.

- Se evalúa con una tasa de descuento de $14 \%$.
- La equivalencia de cambio en unidades monetarias es $1 \mathrm{UF}=\$ 13.625$.

- El interés del capital solicitado a una entidad privada es del $12 \%$ anual.

- Reintegro, el préstamo se cancela en 15 años; con dos años de gracia después de iniciada la operación comercial.

- Precio de compra de la materia prima en mercado playa es de \$500/cangrejo vivo.

- Los precios de venta considerados de acuerdo a símiles en mercado internacional son:

Cangrejo trozado $\$ 5.478 /$ Kilo

$(80 \%$ de precio de mercado $=$ U\$ 16,5/kilo $)$.

Carne de cangrejo: $\$ 4.784 /$ kilo

$(80 \%$ del precio del mercado $=$ U\$ $14,3 /$ kilo $)$.

Depreciación: La depreciación es calculada en forma lineal, estableciéndose un valor residual del 10\% de los valores originales para construcciones, maquinarias y equipos. De este modo, el costo total por concepto de depreciación alcanza los \$7.782.384 anuales, de acuerdo al siguiente detalle:

\begin{tabular}{|lccc|}
\hline Item & Costo & Período de depreciación & Costo dedepreciación \\
Construcción & $\$ 30.130 .000$ & 15 años & $\$ 1.807 .800$ \\
Maquinaria & $\$ 42.532 .950$ & 10 años & $\$ 3.827 .966$ \\
Materiales & $\$ 10.733 .092$ & 5 años & $\$ 2.146 .618$ \\
Total & & & $\$ 7.782 .384$ \\
\hline
\end{tabular}

Costo de producción: Para una producción anual de 14,4 ton de cangrejo trozado y de 33,6 ton de carne de cangrejo, los costos de producción son los siguientes:

\begin{tabular}{|lcc|}
\hline Item & Costo & Observaciones \\
Materia prima & $\$ 96.000 .000$ & - \\
Insumos & $\$ 15.794 .075$ & - \\
Servicios & $\$ 3.623 .544$ & - \\
Fuerza laboral & $\$ 17.200 .000$ & - \\
Reparaciones y mantención & $\$ 2.126 .648$ & $5 \%$ del costo de maquinarias \\
Seguro & $\$ 1.506 .500$ & $5 \%$ del costo de la planta \\
Costo de producción & $\$ 136.250 .767$ & - \\
Depreciación & $\$ 7.782 .384$ & - \\
Interés & $\$ 14.700 .701$ & - \\
Costo grueso de producción & $\$ 158.733 .852$ & \\
\hline
\end{tabular}

Ingresos: Los ingresos anuales por concepto de ventas de los productos de cangrejo dorado congelado, alcanzan un total de \$238.421.760, según el siguiente detalle:

\begin{tabular}{|lccc|}
\hline Producto & Cantidad (ton) & Precio unitario & Ingresos \\
Cangrejo trozado & 14,4 & $\$ 5.478 .400$ & $\$ 78.888 .960$ \\
Carne de cangrejo & 33,6 & $\$ 4.748 .000$ & $\$ 159.532 .800$ \\
Total Ingresos & & & $\$ 238.421 .760$ \\
\hline
\end{tabular}




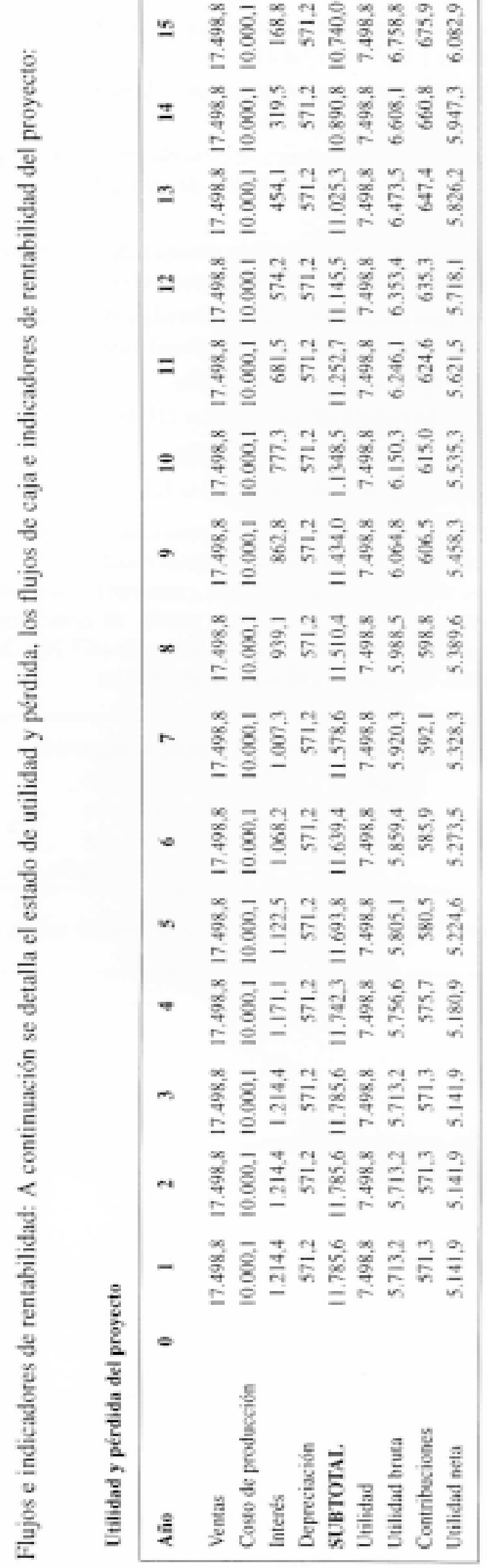

\begin{tabular}{|c|c|c|c|c|c|c|c|}
\hline$\cong$ & $\frac{\infty}{\frac{8}{b}}$ & $\frac{q}{\tilde{n}} \underset{b}{\vec{d}} \overline{\mathrm{d}}$ & $\frac{g}{\frac{n}{n}}$ & & 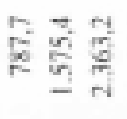 & $\frac{9}{8}$ & 马 \\
\hline \pm & $\frac{\pi}{8}$ & 急学 & $\frac{\frac{y}{g}}{\frac{d}{8}}$ & & $\begin{array}{l}25 \\
5 \frac{2}{2} \\
2\end{array}$ & $\begin{array}{l}\infty \\
\frac{1}{2} \\
\dot{d}\end{array}$ & 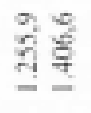 \\
\hline$=$ & $\begin{array}{l}8 \\
\text { g } \\
\dot{b}\end{array}$ & 동 & $\begin{array}{l}\overline{5} \\
\frac{9}{5}\end{array}$ & & 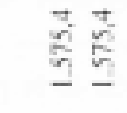 & बे & 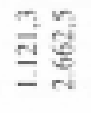 \\
\hline$\simeq$ & $\frac{g}{\sigma}$ & $\frac{c y}{\bar{n}} \frac{\bar{\alpha}}{n}$ & $\begin{array}{l}y \\
y \\
z \\
\approx\end{array}$ & & $\begin{array}{l}2 \\
\frac{2}{2} \\
\frac{5}{2}\end{array}$ & 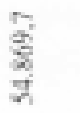 & 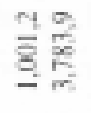 \\
\hline$=$ & $\begin{array}{l}\text { ?2 } \\
\text { है } \\
\text { जी }\end{array}$ & $\frac{4}{5} \frac{n}{n}$ & $\frac{\vec{r}}{\frac{\pi}{n}}$ & & 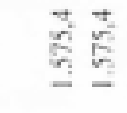 & $\frac{q}{\frac{\vartheta}{n}}$ & 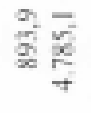 \\
\hline$\triangleq$ & $\begin{array}{l}\frac{0}{3} \\
\frac{8}{4}\end{array}$ & $\frac{y}{F} \underset{z}{m}$ & $\frac{r}{8}$ & & 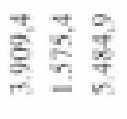 & $\frac{\overrightarrow{2}}{2}$ & $\begin{array}{l}40 \\
20 \\
20 \\
2\end{array}$ \\
\hline$=$ & $\frac{\infty}{\stackrel{B}{g}}$ & $\frac{\pi}{n} \frac{\pi}{\frac{6}{7}}$ & $\frac{\ddot{g}}{\stackrel{y}{q}}$ & & $\begin{array}{l}3 \pm \\
\frac{2}{2}\end{array}$ & $\begin{array}{l}\frac{9}{3} \\
\frac{3}{4} \\
7\end{array}$ & $\frac{0}{2} \frac{c}{g}$ \\
\hline$\propto$ & $\begin{array}{l}\text { है } \\
\text { हi }\end{array}$ & $\frac{4}{5} \frac{0}{b}$ & $\frac{\mathrm{g}}{\mathrm{g}}$ & & $\begin{array}{l}\vec{z} \\
\text { E. } \\
=-\end{array}$ & $\frac{\sqrt{\frac{\gamma}{2}}}{\frac{9}{9}}$ & $\frac{3}{2}$ \\
\hline$r$ & $\frac{a}{3}$ & $\frac{y}{n} \frac{\pi}{2}$ & $\stackrel{\infty}{\stackrel{p}{F}}$ & & $\begin{array}{l}5 \\
6 \\
5\end{array}$ & $\begin{array}{l}\overrightarrow{2} \\
\frac{2}{5} \\
i \\
i\end{array}$ & $\begin{array}{l}\bar{z} \\
x \\
\frac{x}{2}\end{array}$ \\
\hline 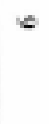 & $\frac{\overline{d i}}{\bar{y}}$ & $\frac{y}{5} \frac{2}{v}$ & $\frac{\tilde{c}}{\tilde{c}}$ & & $\frac{\pi}{2} \frac{4}{2}$ & $\frac{m}{\frac{\pi}{4}}$ & $\stackrel{9}{g} \frac{9}{\infty}$ \\
\hline in & $\begin{array}{l}n \\
\frac{n}{2} \\
\text { हi }\end{array}$ & 동 & $\frac{r}{2}$ & & 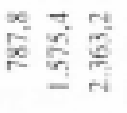 & $\frac{\overline{c i}}{\bar{n}}$ & $\begin{array}{l}\frac{a}{2} \\
\frac{1}{y} \frac{5}{5} \\
\infty\end{array}$ \\
\hline+ & $\begin{array}{l}\infty \\
\text { s.j } \\
\text { o. }\end{array}$ & $\frac{y}{5} \frac{g}{x}$ & $\frac{\vec{q}}{\frac{\vec{q}}{2}}$ & & $\begin{array}{l}\ddot{2} \\
\underline{2} \\
-2\end{array}$ & $\frac{2}{2}$ & $\frac{7}{7} \frac{7}{2}$ \\
\hline$m$ & 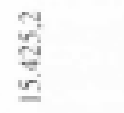 & $\frac{y}{5} \frac{a}{v}$ & $\frac{y}{\frac{y}{m}}$ & & 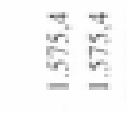 & $\begin{array}{l}\infty \\
\text { di } \\
\underline{0} \\
\underline{2}\end{array}$ & $\frac{7}{9} \frac{2}{2}$ \\
\hline$e$ & $\frac{\bar{d}}{\bar{a}}$ & $\frac{n}{5} \frac{a}{n}$ & $\begin{array}{l}\bar{v} \\
\vec{q} \\
\underline{v}\end{array}$ & & & $\begin{array}{l}\bar{z} \\
\text { हुँ }\end{array}$ & $\begin{array}{l}\infty \\
\equiv \\
\equiv\end{array}$ \\
\hline- & $\stackrel{0}{8}$ & $\frac{n}{5} \frac{\theta}{\frac{\pi}{q}}$ & $\frac{\vec{a}}{\bar{r}}$ & & & $\frac{\bar{d}}{2}$ & $\stackrel{\cong}{\underline{\Xi}}$ \\
\hline$=$ & $\stackrel{0}{\underline{g}}$ & & $\frac{a}{\frac{0}{2}}$ & $\frac{\infty}{\frac{a}{6}}$ & $\frac{\infty}{\mathrm{g}}$ & $\stackrel{8}{8}$ & 总 \\
\hline$\stackrel{g}{2}$ & 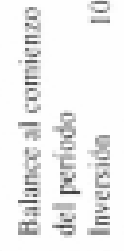 & 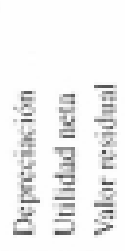 & 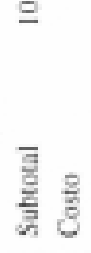 & 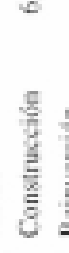 & 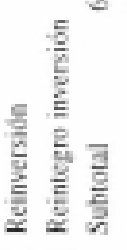 & 帝 & 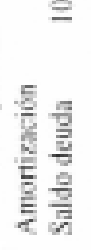 \\
\hline
\end{tabular}


Punto de equilibrio: El punto equilibrio es el nivel de producción en el cual no se producen pérdidas ni ganancias, se expresa en $\mathrm{kg} / \mathrm{año}$. En el proyecto, corresponde a una producción de $3.450,31 \mathrm{~kg}$ de cangrejo trozado y de $8.023,097 \mathrm{~kg}$ de carne de cangrejo. En términos de captura, ello significa un total de 45.928 ejemplares de cangrejo al año.

Análisis de sensibilidad: Para la verificación de la dependencia de la viabilidad del proyecto, se simuló la actividad financiera con respecto a los factores críticos, como son el precio de venta en el mercado internacional del producto (PM) y el precio de playa de la materia prima (PP), con respecto al rendimiento por trampa que se pudiese obtener en la actividad. Es así como se hizo disminuir el primer factor en un 10 , 20, 30 y 40\%, mientras que el segundo se disminuyó en un $20 \%$ y se aumentó en un 20 y $40 \%$. Los resultados de la evaluación se presentan a continuación:

Proyección pesimista (rendimiento $4 \mathrm{~kg} /$ trampa):

\begin{tabular}{|c|c|c|c|c|c|c|c|c|c|c|}
\hline \multirow[b]{2}{*}{ PP } & \multicolumn{2}{|c|}{$P M * 0,6$} & \multicolumn{2}{|c|}{ PM * 0,7 } & \multicolumn{2}{|c|}{$P M+0,8$} & \multicolumn{2}{|c|}{$\mathrm{PM} * 0,9$} & \multicolumn{2}{|c|}{ PM } \\
\hline & $\begin{array}{l}\text { VAN } \\
\text { (UF) }\end{array}$ & $\begin{array}{l}\text { TIR } \\
(\%)\end{array}$ & $\begin{array}{l}\text { VAN } \\
\text { (UF) }\end{array}$ & $\begin{array}{l}\text { TIR } \\
(\%)\end{array}$ & $\begin{array}{l}\text { VAN } \\
\text { (UF) }\end{array}$ & $\begin{array}{l}\text { TIR } \\
(\%)\end{array}$ & $\begin{array}{l}\text { VAN } \\
\text { (UF) }\end{array}$ & $\begin{array}{l}\text { TIR } \\
(\%)\end{array}$ & $\begin{array}{l}\text { VAN } \\
\text { (UF) }\end{array}$ & $\begin{array}{l}\text { TIR } \\
(\%)\end{array}$ \\
\hline$\$ 400$ & $-2.694,1$ & $-3,8$ & $6.104,6$ & 30,8 & $15.173,3$ & 52,1 & $24.241,1$ & 72,3 & 33.310 .7 & 91,8 \\
\hline$\$ 500$ & $-9.521,1$ & $-4,2$ & $.452,4$ & 12,6 & 8.616 .3 & 36,0 & $17.684,1$ & 56,2 & $26.753,7$ & 75,4 \\
\hline$\$ 600$ & $-16.078,0$ & $-7,6$ & $-7,009,3$ & $-1,4$ & 2.059 .4 & 19,6 & 11.127 .2 & 40,8 & $20.196,8$ & 59,9 \\
\hline$\$ 700$ & $-22.634,9$ & $-13,8$ & $-13.566,2$ & $-5,9$ & $-4.497,5$ & -0.4 & 4.570 .3 & 25,4 & $13.639,9$ & 45,1 \\
\hline
\end{tabular}

Situación normal (rendimiento $6 \mathrm{~kg} /$ trampa):

\begin{tabular}{|c|c|c|c|c|c|c|c|c|c|c|}
\hline \multirow[b]{2}{*}{ PP } & \multicolumn{2}{|c|}{$\mathrm{PM} * 0,6$} & \multicolumn{2}{|c|}{$\mathrm{PM} * 0,7$} & \multicolumn{2}{|c|}{$P M=0,8$} & \multicolumn{2}{|c|}{$P M=0,9$} & \multicolumn{2}{|c|}{ PM } \\
\hline & $\begin{array}{l}\text { VAN } \\
\text { (UF) }\end{array}$ & $\begin{array}{l}\text { TIR } \\
(\%)\end{array}$ & $\begin{array}{l}\text { VAN } \\
\text { (UF) }\end{array}$ & $\begin{array}{l}\text { TIR } \\
(\xi)\end{array}$ & $\begin{array}{l}\text { VAN } \\
\text { (UF) }\end{array}$ & $\begin{array}{l}\text { TIR } \\
(\%)\end{array}$ & $\begin{array}{l}\text { VAN } \\
\text { (UF) }\end{array}$ & $\begin{array}{l}\text { TIR } \\
\text { (\%) }\end{array}$ & $\begin{array}{l}\text { VAN } \\
\text { (UF) }\end{array}$ & $\begin{array}{l}\text { TIR } \\
(\%)\end{array}$ \\
\hline$\$ 400$ & $5.080,8$ & 27,2 & 16.755 .9 & 53,2 & $28,487,5$ & 78.2 & $40.939,1$ & 102,4 & 53.030 .7 & 126.2 \\
\hline$\$ 500$ & $4.077,1$ & 1,2 & $8.020,1$ & 33,2 & $17.538,6$ & 48,9 & 32.331 .7 & 82.1 & $44.288,1$ & 104.8 \\
\hline$\$ 600$ & $-12.404,4$ & -5.1 & $-729,2$ & 12,1 & $11.362,3$ & 39,2 & $23,453,9$ & 62.7 & 35.545 .5 & 85,1 \\
\hline$\$ 700$ & $-21,147,0$ & $-11,1$ & -9.741 .8 & $-4,3$ & 2.619 .8 & 20,1 & $14.711,4$ & 44,6 & 26.803 .0 & 66.7 \\
\hline
\end{tabular}

Proyección optimista (rendimiento $8 \mathrm{~kg} /$ trampa):

\begin{tabular}{|c|c|c|c|c|c|c|c|c|c|c|}
\hline \multirow[b]{2}{*}{ PP } & \multicolumn{2}{|c|}{$P M * 0,6$} & \multicolumn{2}{|c|}{$P M=0,7$} & \multicolumn{2}{|c|}{$P M * 0,8$} & \multicolumn{2}{|c|}{$P M * 0,9$} & \multicolumn{2}{|c|}{ PM } \\
\hline & $\begin{array}{l}\text { VAN } \\
\text { (UF) }\end{array}$ & $\begin{array}{l}\text { TIR } \\
(\%)\end{array}$ & $\begin{array}{l}\text { VAN } \\
\text { (UF) }\end{array}$ & $\begin{array}{l}\text { TIR } \\
\text { (\%) }\end{array}$ & $\begin{array}{l}\text { VAN } \\
\text { (UF) }\end{array}$ & $\begin{array}{l}\text { TIR } \\
\text { (\%) }\end{array}$ & $\begin{array}{l}\text { VAN } \\
\text { (UF) }\end{array}$ & $\begin{array}{l}\text { TIR } \\
(\%)\end{array}$ & $\begin{array}{l}\text { VAN } \\
\text { (UF) }\end{array}$ & $\begin{array}{l}\text { TIR } \\
\text { (ङ) }\end{array}$ \\
\hline$\$ 400$ & $12.292,7$ & 42,0 & 27.407 .2 & 71,9 & $45.521,7$ & 100,5 & 57.634 .8 & 128,5 & $75,750.7$ & 156,1 \\
\hline$\$ 500$ & $1.364,5$ & 17,3 & $16.479,0$ & 48,6 & $31.593,5$ & 76.5 & $46.706,5$ & 103,4 & $61.822,5$ & 129,8 \\
\hline$\$ 600$ & $-9.563,7$ & $-4,2$ & $5.550,8$ & 26,1 & $20.665,3$ & 54,7 & $35.778,3$ & 80.5 & 50.894 .3 & 106,0 \\
\hline$\$ 700$ & $-20.491,9$ & $-11,0$ & $-5,377,4$ & -0.4 & $9.737,1$ & 33,4 & $24.850,1$ & 59.5 & $39.966,1$ & 84,2 \\
\hline
\end{tabular}


Se verificó los cambios del valor actual neto variando la tasa de descuento, obteniéndose los siguientes resultados:

\begin{tabular}{|cc|}
\hline Tasa de descuento $(\%)$ & VAN $($ UF $)$ \\
10 & 26.646 \\
12 & 23.093 \\
14 & 20.105 \\
16 & 17.571 \\
18 & 15.405 \\
\hline
\end{tabular}

\section{CONCLUSIONES}

De acuerdo a los resultados obtenidos, se pueden destacar los siguientes aspectos:

1. Las instalaciones en planta permitirían usos alternativos si ocurriesen complicaciones en la plaza de mercado o se derivase el esfuerzo de la flota pesquera artesanal del archipiélago a otra pesquería. 2. La planta en proyecto alcanzaría una producción de 14,4 ton anuales de cangrejo trozado (pinzas y patas), cocido y congelado sin caparazón en porciones de $1 / 2$ y $3 / 4$ lb y 33,6 ton anuales de carne de cangrejo, cocida y congelada en bloques de 1 y $2 \mathrm{lb}$.

3. Las condiciones financieras de la operación hacen al proyecto altamente sensible tanto a los precios de transacción en el mercado de los productos finales, como al precio playa para la obtención de la materia prima. Por ello se dimensionó tanto la planta como la fracción de la flota a dedicar a la pesquería bajo un criterio conservador, que adicionalmente cubre imprevistos que aseguran la rentabilidad de proyecto. Cabe señalar, que gran proporción de la inversión se debe al capital de trabajo destinado a la compra de materia prima, en valores que permiten al sector artesanal absorber las inversiones en innovaciones tecnológicas requeridas para la implementación de la nueva pesquería.

4. Se calculó para el presente proyecto, un retorno sobre la inversión de 1,73; una tasa interna de retorno del 48,91\% y un valor actual neto de UF 17.538,64 equivalentes a $\$ 238.963 .970$. Por otro lado, de acuerdo a la sensibilización realizada, existe una probabilidad del 73,3\% de éxito de la inversión.

\section{REFERENCIAS}

Arana, P. 2000a. Pesca exploratoria con trampas alrededor de las islas Robinson Crusoe y Santa Clara, archipiélago de Juan Fernández, Chile. Invest. Mar., Valparaíso, 28: 39-52.

Arana, P. 2000b. Estimación de abundancia y biomasa del cangrejo dorado (Chaceon chilensis) en el archipiélago de Juan Fernández, Chile. Invest. Mar., Valparaíso, 28: 53-68.

Arana, P. y R. Vega. 2000. Pesca experimental del cangrejo dorado (Chaceon chilensis) en el archipiélago de Juan Fernández, Chile. Invest. Mar., Valparaíso, 28: 69-81.

Cifuentes, A. y J. Quiñinao. 2000. Composición y vida útil como producto cocido del cangrejo dorado (Chaceon chilensis) proveniente del archipiélago de Juan Fernández, Chile. Invest. Mar., Valparaíso, 28: 195-202.

Arana y S. Ziller. 1985. Antecedentes generales sobre la actividad pesquera realizada en el archipiélago de Juan Fernández. En: P. Arana (ed.). Investigaciones Marinas en el Archipiélago de Juan Fernández. Esc. Ciencias del Mar, UCV, Valparaíso, 125-152.

Sapag, N. y R. Sapag. 1993. Preparación y evaluación de proyectos. Segunda Edic., Mc Graw-Hill Interamericana de México, S.A. de C.V., 389 pp.

Weston, J. y T. Copeland. 1988. Finanzas en administración. Octava Edic., Mc Graw-Hill Interamericana de México, S.A. de C.V., 576 pp. 